\title{
The Electric Simulation of 3D Pixel Detector with Low Noises
}

\author{
Qinwen Guo ${ }^{1,2,3, a}$,Qiang Long ${ }^{1,2,3, b}$, Zhongliang Long ${ }^{1,2,3, c}$,and Zheng Li ${ }^{1,2,3, *}$
}

\author{
${ }^{1}$ School of Materials Science and Engineering, Xiangtan University, Xiangtan 411105, China \\ ${ }^{2}$ National-Provincial Laboratory of Special Function Thin Film Materials, and School of Materials \\ Science and Engineering, Xiangtan University, Hunan 411105, China \\ ${ }^{3}$ Center for Semiconductor Particle and photon Imaging Detector Development and Fabrication, \\ Xiangtan University, Xiangtan 411105, China \\ aguoqinwen@hotmail.com, 미1771297308@163.com, ’hlong97@163.com, ${ }^{*}$ lizheng@xtu.edu.cn
}

Key words: pixel detector, low noises, low capacitance

Abstract. The aim of this paper is to reduce the detector electronic noises by reducing the detector capacitance. We designed a new type of electrode that uses a geometrical shape different from conventional detector effective surface area. Main detector electric characteristics, including electric field, electric potential, and electron concentration have been simulated. Through these simulations, detector full depletion voltage $\left(V_{f d}\right)$ has been obtained. It has been found that values of $V_{f d}$ in our new detectors is bigger than that in a detector with conventional electrode shape. The simulated detector capacitance is smaller than that in a detector with conventional electrode shape.

\section{Introduction}

The pixel detector is a kind of device that transforms the radiation signal into an electrical signal, which is based on the Compton-Debye effect of the interaction between light radiation and matter. Silicon photonics pixel detector has characteristic of high position resolution. Nowadays, the semiconductor silicon detector has been used as a vertex and track detector almost in every large high-energy physics laboratory, such as the LHC ATLAS detector in Europe [1], in which the closest to the center of the beam is the pixel detector. However, in the process of detecting the photon, the useful signal and the useless noise signal can both be measured, which will greatly reduce the signal to noise ratio $(S / N)$ [2]. The signal to noise ratio $(S / N)$ is a key parameter of high performance detector, and it is an important index to test whether a detector is available. With the development of science and technology, higher demand for the precision of the device has been put forward. Therefore, the noise of the detector has become a big obstacle for increasing the accuracy of pixel detectors. What's more, it can cause the signal distortion and affect detector accuracy of measurement. Reducing the detector noise is always one of the main tasks for the development of detectors. Detector noise has two sources, including the capacitance and the leakage current. This paper will focus on the reduction of pixel detector capacitance, because it not only increases the noise, but also directly affects the crosstalk of the detector's work.

The anode and cathode of conventional pixel detectors are completely covered by the metal electrode, and the larger effective electrode area makes the capacitance of detector larger [3]. The large capacitance has a significant impact on detector accuracy, which seriously affects the performance of the detector. However, the smaller effective electrode area is likely to introduce dead region. In this work, we proposed an electrode that reduced the effective area, while maintained the sensitive volume unchanged. In order to obtain a relatively uniform electric field potential, the electrode we proposed had symmetry. We have simulated this structure by semiconductor device simulation software (silvaco TCAD), and have obtained the electric field 
distribution, the electric potential distribution, the capacitance and full depletion voltage. Compared with the capacitance and full depletion voltage of conventional pixel detector, it will provide reliable references for the design of silicon pixel photon detector. In this paper, we mainly present a kind of newly designed electrode shape: Ginkgo leaf shape.

\section{Structure of new design pixel detector}

We used silvaco TCAD simulation tool to simulate ginkgo leaf shape. Shown in Fig. 1a and 1b are a single cell of the ginkgo leaf shape. The overall structure of the unit is shown in Fig. 1a. Fig. $1 \mathrm{~b}$ is the accurate size of the ginkgo leaf shape (two dotted lines in Fig. 1b represent the sections of two specific locations, and this article will discuss the electrical properties of these two special locations). In the simulation of the structure of a unit cell, the bulk silicon is n-type high resistivity silicon doped with phosphorus of $8 \times 10^{11} \mathrm{~cm}^{-3}$. The thickness $\mathrm{d}$ of the detector is $500 \mu \mathrm{m}$. There are $1 \mu \mathrm{m}$ thick $\mathrm{p}^{+}$-implanted areas (boron, the peak concentration is $1 \times 10^{19} \mathrm{~cm}^{-3}$, only under the ginkgo leaf shape areas) on the front surface and an $1 \mu \mathrm{m}$ thick $\mathrm{n}^{+}$-implanted layer on the backside surface. On the front surface, $\mathrm{p}^{+}$-implanted areas are covered by 1 um thick aluminum to form the ginkgo leaf shape cathodes. On the bottom (the backside), the $\mathrm{n}^{+}$-implanted layer (phosphorus, the peak concentration is $1 \times 10^{19} \mathrm{~cm}^{-3}$ ) is covered by $1 \mathrm{um}$ thick aluminum acting as the anode. Cathodes are separated by a silicon dioxide layer with an oxide charge density of $4 \times 10^{11} \mathrm{~cm}^{-3}$. $\mathrm{SiO}_{2}$ layer is very stable and has strong chemical inertness, and not easy to react with other substances. $\mathrm{SiO}_{2}$ layer is almost a perfect dielectric layer, and it will be isolated from different small electrode, reducing the crosstalk among pixels. In this study, the design of the electrode shape is mainly designed according to the theory that pixel detector can be full depleted at a reasonable voltage, when distance between two neighboring $\mathrm{p}^{+}$-implants of ginkgo leaf shape is $233 \mu \mathrm{m}$ [4].

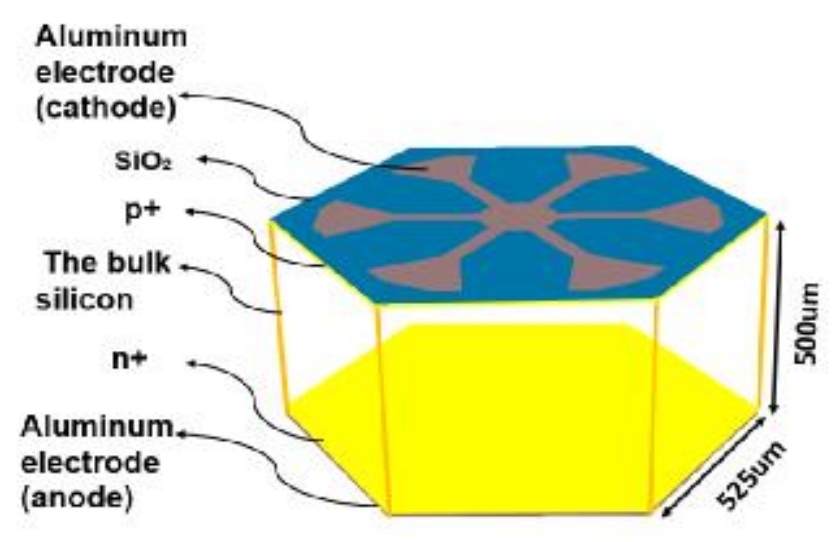

Fig. 1. (a) Structure of the ginkgo leaf shape.

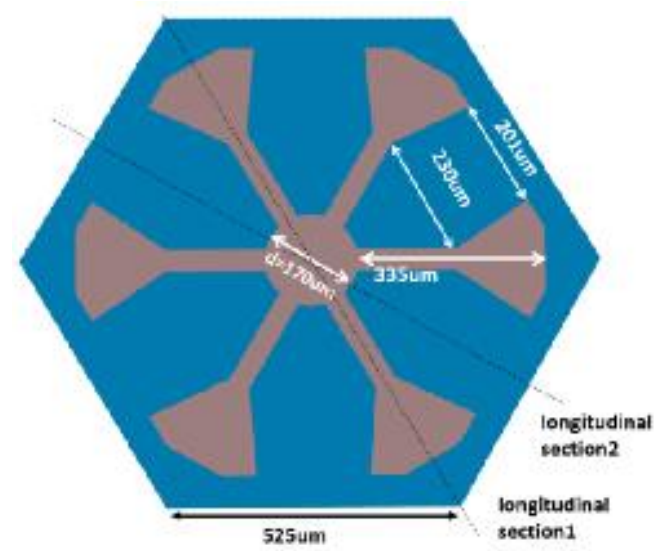

(b) Accurate size of the ginkgo leaf

\section{Full depletion voltage}

Fig. 2a shows the I-V curve of conventional pixel detector. We can draw a conclusion that full depletion voltage of the conventional pixel detector with $500 \mu \mathrm{m}$ thick bulk is $160 \mathrm{~V}$. Fig. $2 \mathrm{~b}$ shows the distribution of electron's density of the ginkgo leaf shape pixel detector at $160 \mathrm{~V}$. As is shown in the Fig. $2 b$, the bulk is not completely depleted. After the simulation, we obtained that the full depletion voltage was $250 \mathrm{~V}$. What are shown in Fig. $2 \mathrm{c}$ and $2 \mathrm{~d}$ are the electron concentration profiles of 2D cut plane of longitudinal section 1 and longitudinal section2 (as the Fig.1b shows). In Fig $.2 \mathrm{~d}$, the $\mathrm{p}^{+}-$-implanted area and the electrode cover area are the smallest, but we can find that the region of the bulk is completely depleted at $250 \mathrm{~V}$. 


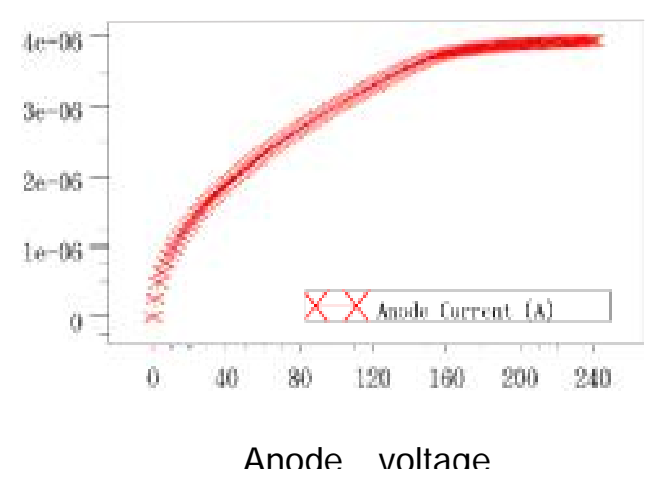

Fig. 2 (a) The I-V curve of conventional pixel detector.

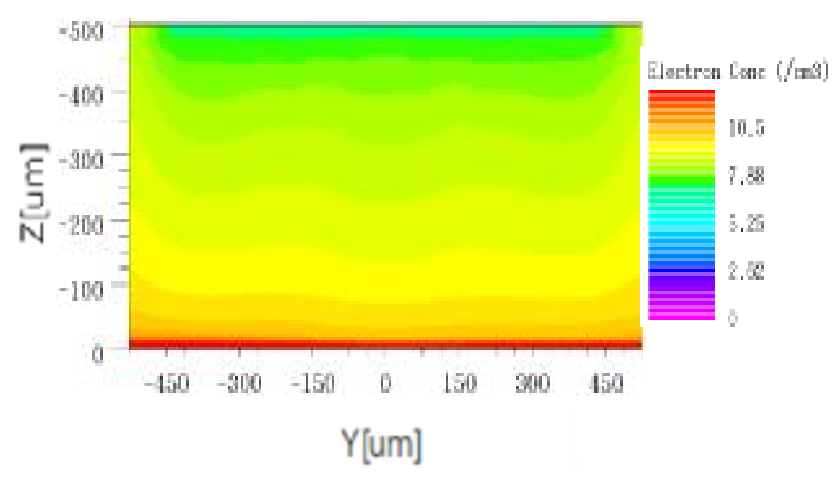

Fig. 2. (c) Electron concentration in the longitudinal section 1 at $250 \mathrm{~V}$

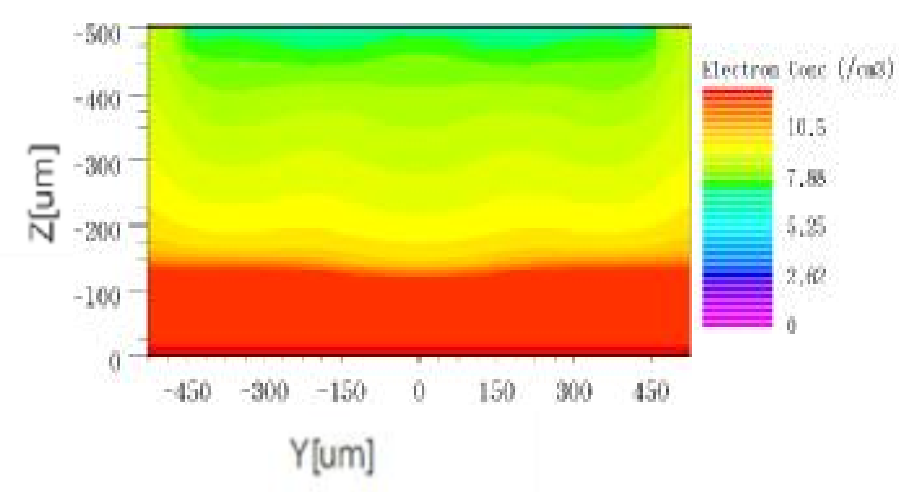

Fig. 2 (b) Electron concentration at160V

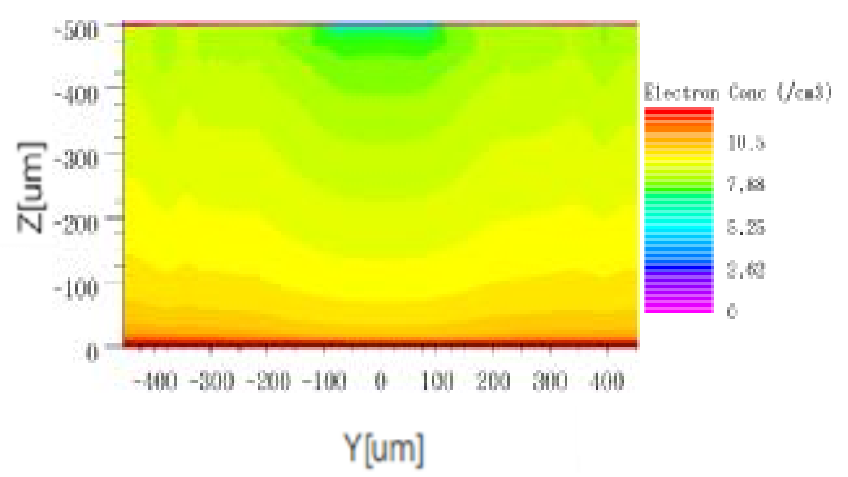

Fig. 2(d) Electron concentration in the longitudinal section 2 at $250 \mathrm{~V}$

\section{Electric potential characteristics}

Shown in Fig. 3a and $3 \mathrm{~b}$ are the electric potential distributions of the ginkgo leaf shape in 2D section views (longitudinal section 1 and longitudinal section2). The bias voltage is set at $250 \mathrm{~V}$.

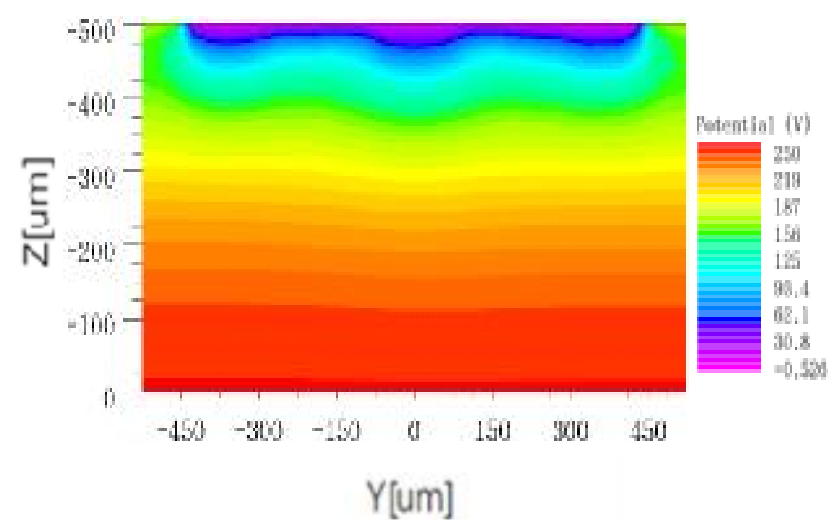

Fig. 3. (a) Electric field profile in the longitudinal section 1 at $250 \mathrm{~V}$

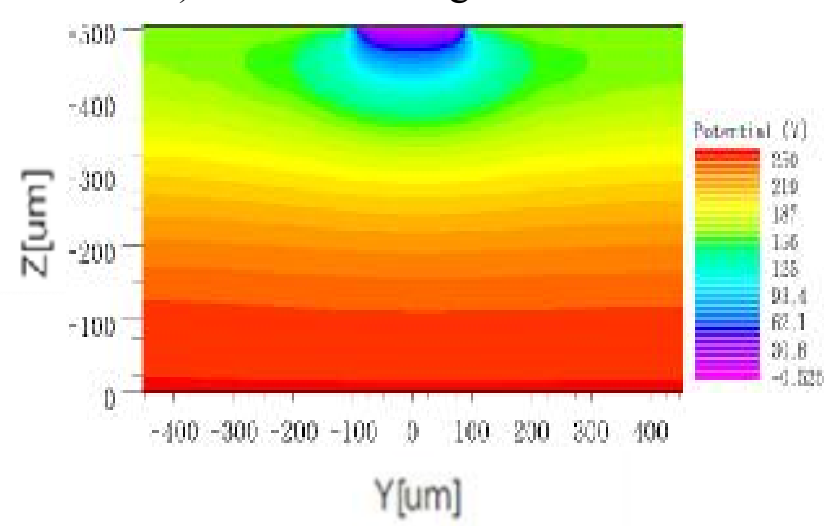

(b) Electric field profile in the longitudinal section 2 at $250 \mathrm{~V}$

\section{Electric field characteristics}

Fig. $4 \mathrm{a}$ and $4 \mathrm{~b}$ (longitudinal section 1 and longitudinal section2) show the results of the electric field of the ginkgo leaf shape detectors at the bias voltage of $250 \mathrm{~V}$. The distribution of electric field is gentle in the whole area, with no obviously high electric field district existed, and it is not easy to generate local electric-breakdown. 

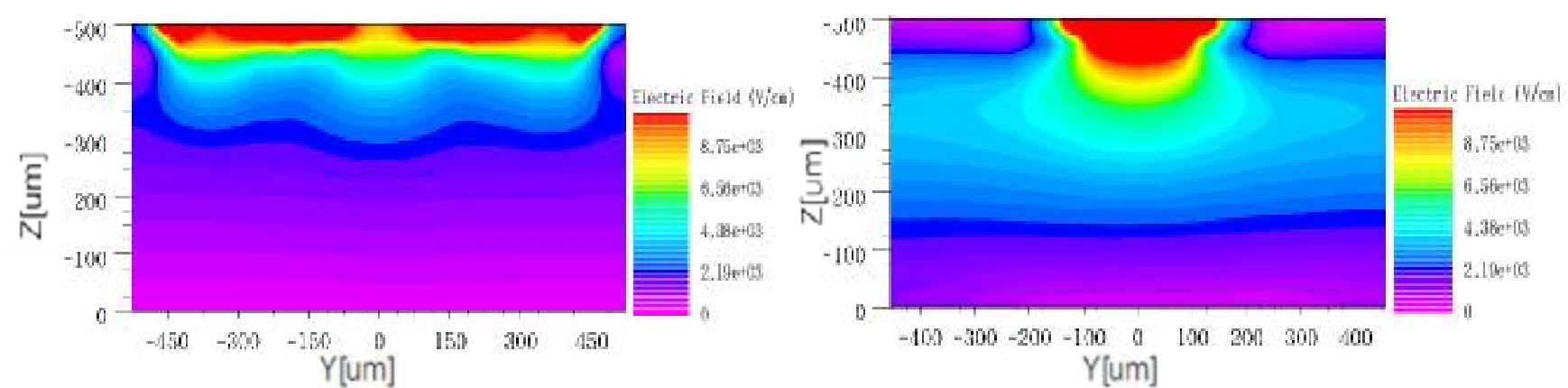

Fig. 4. Electric field profile(a) in the longitudinal section 1 at $250 \mathrm{~V}$ (b) in the longitudinal

\section{Capacitance-voltage characteristics}

Capacitance is a sensitive factor in the silicon detector because it directly affects the noise and crosstalk of the detector's work [5]. The capacitance-voltage characteristics are obtained by using a small signal analysis (AC analysis, frequency is $1 \times 10^{6} \mathrm{~Hz}$ ) [6]. The capacitance of the newly designed detectors (ginkgo leaf shape) is plotted in Fig. 5. They are reduced by about 1/12 as compared to conventional pixel detectors with cathode electrode filling the whole cell area.

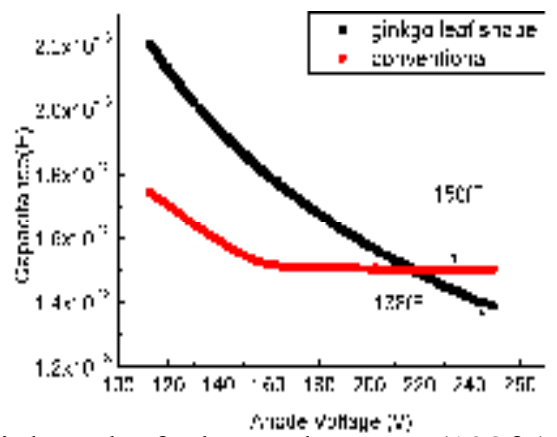

Fig. 5. The capacitance of ginkgo leaf shape detector (138fF) comparing with the conventional

\section{Conclusion}

Through 3D simulations and calculations, we have found that the capacitance of ginkgo leaf shape structure is $138 \mathrm{fF}$. while hexagonal structure of conventional capacitance is $150 \mathrm{fF}$. Due to the decrease of effective electrode area, the capacitance of the ginkgo leaf shape structure compared with conventional silicon pixel detector (hexagonal) has reduced by nearly $1 / 12$ of the capacitance. By extracting the simulation plot of electron concentration, depleted voltage of the ginkgo leaf shape structure is $250 \mathrm{~V}$. Compared with the conventional pixel detectors, due to the decrease of the effective electrode area, depletion voltage has increased. Therefore, the balance between capacitance and voltage can be adjusted according to the requirements of practical application.

\section{References}

[1] J.N. Jackson, Nuclear Instruments and Methods Section A 541 (2005) 89.

[2] Miroslav Havra'nek, et al., Nuclear Instruments and Methods Section A 714 (2013) 83.

[3] Z. Li, Nuclear Instruments and Methods Section A 612 (2010) 509.

[4] Bo. Xiong, et al., Nuclear Instruments and Methods in Physics Research A 831 (2016) 34-37.

[5] G. Gorfine, et al., Nuclear Instruments and Methods Section A 465 (2001) 70.

[6] J. Chen, et al., Nuclear Instruments and Methods Section A 796 (2015) 34. 